\title{
Erratum: Structure of the quantum spin Hall states in HgTe/CdTe and InAs/GaSb/AISb quantum wells [Phys. Rev. B 91, 035310 (2015)]
}

\author{
P. C. Klipstein \\ (Received 13 April 2016; published 26 May 2016)
}

DOI: 10.1103/PhysRevB.93.199905

In Secs. II and VI, it was incorrectly stated that the edge state wave function $e^{-\sigma_{+}^{\prime} y}-e^{-\sigma_{-}^{\prime} y}$, derived using open boundary conditions (OBCs), must vanish at the singular wave vectors $k_{S(1,2)}^{\prime}$, because $v^{\prime}=0$ and the decay parameters $\sigma_{ \pm}^{\prime}=\mu^{\prime} \pm i v^{\prime}$ are then degenerate. The normalized wave function is in fact given by $\frac{1}{v^{\prime}} \sqrt{\mu^{\prime}\left(\mu^{\prime 2}+v^{\prime 2}\right)}\left\{e^{-\sigma^{\prime}+y}-e^{-\sigma^{\prime}-y}\right\}$, which does not vanish in the limit $v^{\prime} \rightarrow 0$, but rather yields $2 \mu^{\prime \frac{3}{2}} y e^{-\mu^{\prime} y}$. This limiting solution is consistent with the theory of second order differential equations with constant coefficients, where two degenerate solutions exist of the form $e^{-\sigma^{\prime} y}$ and $y e^{-\sigma^{\prime} y}$. The vanishing of the OBC wave function at the singular wave vectors was taken as evidence of its unphysical behavior. Although this interpretation is incorrect since the OBC wave function does not vanish, the unphysical behavior of the OBC edge state dispersions discussed at the end of Sec. II is still a valid argument.

It was recognized in the Note added in proof that for the degenerate solutions of weakly hybridized systems, the interpretation given in Sec. V is also incorrect, because in the absence of disorder it allows two states to exist at small wave vectors, and only one at larger wave vectors, violating the fundamental principle of conservation of eigenvalues when some system parameter is varied. An alternative interpretation was given that overcomes this difficulty, where orthogonal linear combinations of the degenerate states are constructed, such that one of them, $\psi_{C}$, can deform smoothly into the unique physical solution beyond the singular wave vectors. It was stated there that the other solution, $\psi_{S}$, will vanish at the singular wave vectors. Although it does have a limiting value when $v^{\prime} \rightarrow 0$, as for the $\mathrm{OBC}$ wave function, it vanishes at larger wave vectors in the sense that there is only one physical solution into which it cannot deform smoothly. $\psi_{S}$ is therefore also regarded as a spurious solution which does not exist physically at any wave vector.

The following (noncritical) typographical errors should be noted. In Fig. 2, the " $\sigma$ " symbols should be primed while $\mu$ ' and $v^{\prime}$ in the Note added in proof should not. There should be a minus sign in front of $P$ in Eq. (12) and the expressions for $c$ in Eq. (11b) and $R_{ \pm}$in the Note added in proof should be multiplied by 2 . 